\title{
Efectos de la infección y la enfermedad por citomegalovirus en receptores de trasplante renal
}

\section{Effects of cytomegalovirus infection and disease in renal transplant recipients.}

\author{
James Díaz-Betancur, Jorge Enrique Henao, Fabián Alberto Jaimes • \\ Medellín (Colombia)
}

\section{Resumen}

La infección por citomegalovirus (CMV) es una de las más importantes causas de morbilidad en pacientes con trasplante renal.

Objetivo: revisar las manifestaciones de la enfermedad aguda por este virus y sus efectos directos e indirectos sobre injerto y receptor en pacientes con trasplante renal.

Material y métodos: se hizo una revisión sistemática de la literatura en la que se encontraron 40 publicaciones completas relacionadas con el tema.

Conclusiones: además de la enfermedad aguda que se manifiesta con síndrome de mononucleosis o daño tisular, el CMV produce efectos indirectos sobre el injerto y el sistema vascular del receptor que parecen aumentar el riesgo de enfermedad cardiovascular y acortan la supervivencia del trasplante y el receptor. Las estrategias utilizadas en los últimos años han logrado disminuir el efecto nocivo de la enfermedad aguda por CMV, pero se desconoce su impacto sobre los efectos indirectos de la infección latente. (Acta Med Colomb 2012; 37: 131-137)

Palabras clave: citomegalovirus, trasplante renal,
Dr. James Díaz-Betancur: Internista, Hospital San Vicente Fundación. Profesor de Medicina Interna Universidad de Antioquia; Dr. Jorge Enrique Henao Sierra: Internista, Nefrólogo, Hospital San Vicente Fundación. Profesor de Nefrología Universidad de Antioquia; Dr. Fabián Alberto Jaimes Barragán: Profesor de Medicina Interna Universidad de Antioquia. Investigador Asociado, Unidad de Investigaciones Hospital Pablo Tobón Uribe. Medellín (Colombia)

Correspondencia. Dr. James Díaz Betancur E-mail: jasadibe@hotmail.com

Recibido: 23/XI/2011 Aceptado: 20/VIII/2012

\section{Introducción}

El citomegalovirus humano (CMV) es un herpesvirus que puede ser transmitido entre humanos por vía perinatal, contacto sexual (1), sangre y tejidos (2), o por contacto personal estrecho (3). La infección es casi asintomática en los individuos inmunocompetentes, pero en receptores de trasplantes es una de las principales causas de morbilidad y mortalidad. El virus entra a las células hospederas por fusión de su envoltura con la membrana celular o a través de fagocitosis y, al igual que otros herpesvirus, después de la resolución de la infección aguda permanece latente en los tejidos por varios mecanismos que le permiten evitar las defensas del hospedero. Entre estos mecanismos están su capacidad para regular a la baja la expresión del complejo 
mayor de histocompatibilidad clase I y evadir el reconocimiento por los linfocitos T citotóxicos, entre otros (4). Los leucocitos son el principal reservorio del CMV, en particular las células CD13 (5), pero el ADN viral ha sido detectado en monocitos, células dendríticas, megacariocitos y células progenitoras mieloides de la médula ósea (6). La exposición al virus, indicada por la presencia de anticuerpos séricos, oscila entre 40-100 \% en la población adulta en diferentes partes del mundo (7). Esta seroprevalencia varía con la edad (8), la raza y la etnicidad (9) y se correlaciona inversamente con el desarrollo socioeconómico al mostrar las mayores tasas en países africanos y asiáticos en desarrollo (10).

El CMV puede producir infección o enfermedad en el hospedero humano. Se considera infección la detección del virus por serología, técnicas moleculares, o cultivos de tejido, mientras para la enfermedad se requiere de síntomas y signos clínicos o manifestaciones de daño de órgano blanco (11). La infección generalmente ocurre en edades tempranas de la vida y la mayoría de veces es asintomática o se presenta como un síndrome de mononucleosis, aunque en mujeres embarazadas puede llevar a complicaciones graves por infección fetal. La enfermedad en cambio se presenta años más tarde, reflejando una de dos posibilidades: la reactivación del virus latente o la reinfección con una cepa nueva, especialmente en individuos inmunocomprometidos.

Para demostrar la presencia del CMV se pueden utilizar pruebas directas que detectan el microorganismo, sus proteínas o sus ácidos nucleicos, o pruebas indirectas como la serología que miden la respuesta inmune al mismo. La presencia de anticuerpos IgG en el plasma indica exposición pasada al virus y los anticuerpos IgM o el aumento de los títulos IgG infección reciente. El CMV, sus proteínas o sus ácidos nucleicos pueden ser detectados en sangre por varias técnicas como la viremia, la antigenemia y la ADNemia. Además, el virus se puede aislar en cultivos de fluidos y de tejidos pero la técnica tiene varias limitaciones y no se emplea de rutina (12). La detección de antígenos permite acelerar el diagnóstico y específicamente la antigenemia especialmente de pp65 parece correlacionarse con la viremia en pacientes con infección por VIH y trasplantes de órgano sólido (13). También se han utilizado pruebas de amplificación molecular para determinar la carga total de partículas virales, pero al igual que la antigenemia estas pruebas han sido poco estudiadas en pacientes inmunocompetentes (14).

La enfermedad por CMV se presenta con fiebre, leucopenia, o manifestaciones de daño en órganos o tejidos blanco como: neumonitis, hepatitis, enfermedad gastrointestinal, pancreatitis, y menos frecuentemente meningoencefalitis y miocarditis $(11,15,16)$, e incluso se han descrito casos atípicos con hemorragia de tracto digestivo (17). Parece ser que la carga viral se correlaciona con la evidencia clínica de enfermedad, la gravedad de la misma y la respuesta al tratamiento (18-21).

La infección por CMV es una de las más importantes en pacientes con trasplante renal, el virus es altamente prevalen- te en receptores o puede ser transmitido en las transfusiones sanguíneas peritrasplante o en el injerto renal. La exposición previa al virus, indicada por la presencia de anticuerpos en el plasma, está presente en más de dos tercios de donantes y receptores de trasplantes (11) y la administración de medicamentos inmunosupresores para evitar el rechazo al injerto aumenta el riesgo de reactivación del microorganismo (22, 23). Con el propósito de revisar las manifestaciones de la enfermedad aguda por este virus y sus efectos directos e indirectos sobre injerto y receptor en pacientes con trasplante renal se hizo una revisión sistemática de la literatura.

\section{Material y métodos}

Se hizo una búsqueda sensible en las bases de datos MEDLINE, CENTRAL y LILACS con la siguiente estrategia de búsqueda en inglés y español. Pacientes: "kidney transplantation" [MeSH], kidney transplantation[tw], renal transplantation[tw], kidney grafting[tw]. Intervención: "cytomegalovirus" $[\mathrm{MeSH}]$, citomegalovirus[tw]. Desenlaces: "infectious mononucleosis" [MeSH], infectious mononucleosis[tw], "graft survival"[MeSH], graft survival[tw], "death" [MeSH], death[tw], "mortality" [MeSH], "survival" [MeSH], survival[tw], cardiovascular death [tw], cardiovascular mortality[tw], "atherosclerosis" [MeSH], atheroscleroses [tw]. Se revisaron las referencias bibliográficas de los artículos encontrados en estas bases de datos en búsqueda de referencias adicionales. Finalmente se encontraron seis revisiones $(5,24-28)$, una guía de práctica clínica (29) y 33 publicaciones completas de otro tipo (26, 30-62), que fueron utilizadas para la elaboración de este texto.

\section{Prevalencia e incidencia}

Hay discrepancia entre países en los informes de prevalencia e incidencia de la infección-enfermedad por CMV en trasplantados (63). Esto es explicado por factores como la raza, el desarrollo socioeconómico, el tipo de trasplante, los métodos de detección viral utilizados, el régimen y la intensidad del tratamiento inmunosupresor, y el estatus serológico de donante y receptor (5). Los centros de control de enfermedades (CDC, Atlanta) estiman una prevalencia de la infección entre 50-80\% para adultos mayores de 40 años, y se cree que la prevalencia alcanza $66 \%$ entre los donantes y los receptores adultos en Estados Unidos. En un estudio iraní la infección y la enfermedad fueron detectadas en $82.5 \%$ y $25 \%$ de los pacientes, respectivamente (55). En un análisis de 207 trasplantados renales españoles $15.7 \%$ presentaron infección por el virus y de éstos $17.2 \%$ desarrollaron la enfermedad (52). En Latinoamérica se han publicado muy pocos estudios sobre el tema y la mayoría son pequeñas series de pacientes $(25,37,39,40,51,53,54,64)$. Un pequeño estudio colombiano (30) en el que se midieron anticuerpos contra los agentes del síndrome TORCHS a 60 gestantes en Medellín se encontró una seroprevalencia del 66\% para CMV y en otro estudio (31) en 455 muestras en la misma ciudad se encontró un porcentaje de infección de 
$89 \%$ en receptores de trasplante renal, $81 \%$ en donantes intrafamiliares, $73 \%$ en donantes cadavéricos y $74 \%$ en individuos normales.

La mayor incidencia de la enfermedad se da en los primeros seis meses postrasplante $(59,65)$ pero la infección puede ocurrir muchos años después (66). Un estudio de cohorte encontró que $20 \%$ de los pacientes desarrollaron la infección más de un año postrasplante (59). Independientemente de las cifras descritas, se ha demostrado que los receptores seropositivos para CMV tienen mayor incidencia de enfermedad aguda por CMV y de pérdida del injerto, y acarrean mayores costos económicos globales en comparación con los receptores negativos (41).

\section{Implicaciones clínicas}

Los principales efectos del CMV en los receptores de trasplantes son de dos tipos: la enfermedad aguda con signos clínicos como fiebre, síndrome de mononucleosis o daño tisular; y los efectos indirectos de la infección latente sobre el injerto y el sistema inmune del receptor $(67,68)$. Se ha observado que la enfermedad aguda en el postrasplante temprano tiene un efecto perjudicial en la supervivencia del receptor a largo plazo. En un estudio de casi 500 pacientes se detectó antigenemia viral en más de $60 \%$ de los individuos en los primeros 100 días postrasplante, y los pacientes con la enfermedad o la infección asintomática tuvieron un riesgo relativo de mortalidad global de 2.5 y 2.9 veces más, respectivamente, que los pacientes sin antigenemia (42).

Entre los efectos indirectos se ha relacionado el virus con la aparición de otras infecciones (69), enfermedades linfoproliferativas (especialmente las asociadas con el virus Epstein-Barr) (43, 67, 70), daño del injerto y acortamiento de su supervivencia $(43,71)$, aterosclerosis sistémica y aumento en el riesgo de enfermedad cardiovascular. Se ha sugerido que la infección por sí misma es un factor de riesgo independiente para el desarrollo de rechazo del injerto (72, 73), al parecer inducido por vasculopatía y respuesta a lo inmune $(43,71,74)$. Un estudio en 24 candidatos a trasplante renal y sus respectivos donantes del Hospital Universitario San Vicente Fundación en Medellín, Colombia, encontró una fuerte asociación entre rechazo e infección por CMV (32).

Recientemente se demostró que la infección se asocia con fibrosis intersticial y atrofia tubular (75), y hay informes que describen un tipo de glomerulopatía del injerto caracterizada por engrosamiento miointimal e inducida por el virus (76, 77). Aunque el mecanismo exacto permanece incierto, la evidencia sugiere que la infección latente de las células endoteliales promueve el engrosamiento miointimal a través de la migración de células de músculo liso arterial mediada por receptores de quimiocinas codificados por el microorganismo (78). Adicionalmente, el virus aumenta la expresión de receptores "scavenger" y la captación de lipoproteínas de baja densidad oxidadas que favorecen la aterosclerosis sistémica (79). Igualmente, se ha demostrado por distintas técnicas como la reacción en cadena de polimerasa (PCR), la hibridación in situ y la inmunohistoquímica la presencia del virus dentro de las placas ateroscleróticas (80).

Aunque se sabe que los trasplantados renales tienen mayor riesgo de morbilidad y mortalidad cardiovascular prematura (81), el papel del CMV en dicho riesgo es difícil de evaluar; ya que también podría ser atribuido a factores relacionados con la falla renal crónica previa al trasplante como la edad, la hipertensión arterial, el tratamiento con diálisis o el trasplante por sí mismo. Además, se sabe que los receptores de trasplante renal tienen mayor riesgo de desarrollar diabetes mellitus y dislipidemia (82), lo que también podrían influir en la supervivencia del paciente y del injerto. Incluso los medicamentos inmunosupresores utilizados para el trasplante podrían modificar la incidencia y la gravedad de las complicaciones cardiovasculares, como se conoce para los corticosteroides y la ciclosporina que tienen efectos sobre la ganancia de peso, la presión arterial y los lípidos $(83,84)$, y con una asociación con hipertensión arterial que podría influir en la supervivencia de los trasplantados $(85,86)$. Así mismo, el tacrolimus incrementa el riesgo de aparición de diabetes mellitus de novo (87), el sirolimus también puede ser diabetogénico (88), y junto con el everolimus son los mayores inductores de dislipidemia postrasplante (89). Dado lo anterior, la evaluación del impacto real del CMV sobre el riesgo de enfermedad cardiovascular en esta población representa un desafío clínico y metodológico, dado su carácter crónico y los múltiples factores de confusión involucrados.

\section{Estatus serológico y riesgo de enfermedad por CMV}

El principal factor de riesgo conocido para desarrollar enfermedad por CMV es el estatus serológico del donante (D) y el receptor $(\mathrm{R})$ determinado por la presencia de anticuerpos séricos de tipo IgG contra el virus. Se sabe que hay mayor riesgo de enfermar cuando el donante, el receptor o ambos tienen anticuerpos contra el virus $(29,90,91)$. Un pequeño estudio encontró asociación entre la condición serológica D+ y el riesgo de enfermedad, independiente de la serología del receptor (49), pero lo más estudiado para determinar el riesgo de enfermedad por CMV es el binomio D/R. Históricamente, entre 60-80\% de los receptores seronegativos (R-) de donantes seropositivos (D+) desarrollaban la infección (47) y entre 10-15\% presentaban la enfermedad si no recibían ningún tratamiento preventivo $(27,38,41)$. De estos receptores enfermos, $30 \%$ hacían neumonitis con una mortalidad hasta de $15 \%$.

En los grupos serológicos $\mathrm{D}+/ \mathrm{R}+\mathrm{y} \mathrm{D}-/ \mathrm{R}+$ la incidencia de la enfermedad aguda es mucho menor, pero el grupo $\mathrm{D}+/$ $\mathrm{R}+$ tiene la más baja supervivencia de injerto y receptor al tercer año postrasplante $(34,35)$. La explicación para este fenómeno no es clara pero podría ser consecuencia de los efectos indirectos de la infección latente, y se ha sugerido que la inmunidad preexistente en los individuos receptores sea específica contra una cepa y por tanto la cepa "nueva" del receptor no pueda ser controlada (5). Esta idea es respaldada por estudios que han demostrado que la sobreinfección 
durante el embarazo puede producir enfermedad en el feto a pesar de los anticuerpos preexistentes en la madre $(92,93)$. Otra explicación para la pobre supervivencia del injerto en estos pacientes es la localización renal del virus, porque la inmunidad humoral no protege contra las células infectadas y el CMV puede existir en forma viable en riñones normales durante periodos prolongados a pesar de la inmunidad celular (94). En los pacientes D-/R+ puede haber reactivación del virus, la enfermedad se desarrolla hasta en $20 \%$ de los casos pero la progresión a neumonitis y muerte es infrecuente. El grupo D-/R- tiene la menor incidencia de la enfermedad pero también representa el menor número de trasplantados. Un estudio local de 146 parejas donante/receptor encontró que $76 \%$ correspondían al grupo $\mathrm{D}+\mathrm{R}+\mathrm{y}$ sólo $2 \%$ fueron D-/R- (31).

\section{Otros factores de riesgo}

En estudios previos han sido mencionados como posibles factores de riesgo para enfermedad aguda el sexo masculino $(58,73)$, la diabetes mellitus como causa de la insuficiencia renal, la edad mayor a 55 años $(58,95)$ y la terapia de reemplazo renal antes del trasplante (96). También se ha descrito una incidencia mayor de la enfermedad sintomática en pacientes D+/R- con cero compatibilidades de antígenos HLA-DR (90). Con respecto a los medicamentos inmunosupresores los datos son poco claros, pero se ha observado que la evolución permanente de los tratamientos ha influido históricamente sobre el riesgo de la enfermedad por CMV. La exposición a ATG (globulina antitimocítica humana), un inmunosupresor que se utilizaba como tratamiento de inducción y algunas veces como terapia antirrechazo en los 80 , ha sido relacionada con mayor riesgo de enfermedad (97). Así mismo, la reactivación del virus también era común cuando se utilizaba OKT3 en los protocolos anti-rechazo $(98,99)$. El tratamiento con alemtuzumab y tacrolimus incrementa la incidencia de infección cuando se compara con tacrolimus más micofenolato, pero en ninguno de los casos se ha presentado compromiso tisular (100). Según algunos estudios el tratamiento con micofenolato también incrementa la incidencia de CMV $(101,102)$, y otros han demostrado mayor riesgo con las terapias de mantenimiento que incluyen ciclosporina $(50,52)$. En un estudio colombiano en 12 parejas donante/receptor de candidatos a trasplante renal se observó un promedio de IFN $\gamma$ mayor en los pacientes que presentaron enfermedad por CMV pero la diferencia no fue significativa (33).

\section{Prevención}

Se han utilizado múltiples estrategias para reducir la morbilidad, la mortalidad y los costos asociados a la infección por CMV; entre las cuales se cuentan evitar la incompatibilidad serológica, en especial D+/R-, que es una medida poco práctica, administrar tratamiento una vez establecida la enfermedad o utilizar estrategias preventivas o profilácticas. Anteriormente sólo se administraba tratamiento antiviral para la enfermedad sintomática y esto resultaba en una incidencia entre $20-60 \%$ con altas tasas de mortalidad (36), pero el advenimiento de tratamientos profilácticos y de rescate ha mejorado esta situación $(91,103)$ y parece que la profilaxis contra el virus también reduce el riesgo de rechazo $(36,104)$.

Las estrategias más ampliamente utilizadas para disminuir el riesgo de enfermedad aguda son dos: la primera consiste en administrar profilácticamente antivirales a los pacientes con mayor riesgo (estrategia profiláctica), y la segunda en monitorizar periódicamente la viremia para iniciar el tratamiento inmediatamente se detecta el virus (estrategia preventiva). Los metanálisis que han comparado la estrategia preventiva contra el tratamiento estándar y la estrategia preventiva contra la profiláctica no han encontrado diferencias, pero al compararlas contra placebo si se ha observado una reducción significativa en el riesgo de la enfermedad, especialmente para los pacientes D-/R+ (105-107).

Los medicamentos utilizados para la profilaxis son el ganciclovir y el valganciclovir, que han desplazado al aciclovir y a la globulina hiperinmune como el tratamiento de elección (107). A pesar de ser económico y disponible ampliamente, el aciclovir a concentraciones clínicamente alcanzables no tiene actividad contra el virus y los resultados de los ensayos que han evaluado su efectividad para prevenir la enfermedad aguda son contradictorios $(108,109)$, además su utilización no afecta la supervivencia del injerto (48).

Otros agentes antivirales como la globulina hiperinmune contra CMV, el foscarnet y el cidofovir han sido menos estudiados o son nefrotóxicos y se utilizan principalmente cuando se detecta resistencia a ganciclovir $(110,111)$. Alguna evidencia sugiere que el valaciclovir también puede ayudar a prevenir la enfermedad en trasplantados de alto riesgo $(36,112)$, pero se han notificado casos de microangiopatía trombótica con altas dosis del medicamento. Al igual que con el tratamiento de cualquier agente infeccioso, el riesgo de seleccionar cepas resistentes es motivo de preocupación. El fenómeno podría ser más común en binomios $\mathrm{D}+/ \mathrm{R}$ - (113), aunque afortunadamente los casos ha sido pocos y la mayoría en pacientes con trasplante pulmonar (114), con sólo un reporte en un trasplantado renal (115). Como tratamientos alternativos se ha intentado vacunar a los receptores, pero la vacuna estudiada sólo logró reducir la gravedad de la enfermedad sin impedirla (116), por lo que actualmente se encuentran en estudio otras vacunas y nuevos medicamentos (117).

\section{Conclusiones}

Los efectos deletéreos de la enfermedad aguda por CMV en receptores de trasplante renal son bien conocidos. No obstante, resultan más inquietantes los efectos indirectos de la infección latente sobre el injerto y el sistema inmune del receptor que podrían explicar la asociación entre el virus y el acortamiento de la supervivencia del trasplante, el aumento en el riesgo de enfermedad cardiovascular e incluso la menor 
supervivencia del receptor. Las estrategias utilizadas en los últimos años han logrado disminuir el efecto nocivo de la enfermedad aguda por CMV, pero se desconoce su impacto sobre los efectos indirectos de la infección latente, lo que representa un desafío investigativo debido al carácter crónico y multifactorial de estos efectos.

\section{Conflictos de interés}

Los autores declaran no haber recibido financiación de la industria farmacéuticas o de otros patrocinadores para la realización de este manuscrito y no tener relación con compañías que puedan tener un interés económico en la información contenida en este artículo.

\section{Referencias}

1. Jordan MC, Rousseau WE, Noble GR, Steward JA, Chin TD. Association of cervical cytomegaloviruses with venereal disease. N Engl J Med 1973; 288(18): 932-4.

2. Preiksaitis JK, Brown L, McKenzie M. The risk of cytomegalovirus infection in seronegative transfusion recipients not receiving exogenous immunosuppression. J Infect Dis 1988; 157(3): 523-9.

3. Adler SP. Molecular epidemiology of cytomegalovirus: viral transmission among children attending a day care center, their parents, and caretakers. J Pediatr 1988; 112(3): 366-72.

4. Ploegh HL. Viral strategies of immune evasion. Science 1998; 280(5361): 248-53.

5. Brennan DC. Cytomegalovirus in renal transplantation. J Am Soc Nephrol 2001; 12(4): $848-55$

6. Soderberg-Naucler C, Fish KN, Nelson JA. Reactivation of latent human cytomegalovirus by allogeneic stimulation of blood cells from healthy donors. Cell 1997; 91(1): 119-26.

7. Krech U. Complement-fixing antibodies against cytomegalovirus in different parts of the world. Bull World Health Organ 1973; 49(1): 103-6.

8. Klemola E, Kaariainen L. Cytomegalovirus as a possible cause of a disease resembling infectious mononucleosis. Br Med J 1965; 2(5470): 1099-102.

9. Staras SA, Dollard SC, Radford KW, Flanders WD, Pass RF, Cannon MJ. Seroprevalence of cytomegalovirus infection in the United States, 1988-1994. Clin Infect Dis 2006; 43(9): 1143-51.

10. Ho M. Epidemiology of cytomegalovirus infections. Rev Infect Dis 1990; 12 Suppl 7: S701-10.

11. Rubin RH. Infectious disease complications of renal transplantation. Kidney Int 1993; 44(1): 221-36.

12. Gandhi MK, Khanna R. Human cytomegalovirus: clinical aspects, immune regulation, and emerging treatments. Lancet Infect Dis 2004; 4(12): 725-38.

13. Suzuki Y, Israelski DM, Dannemann BR,Stepick-Biek P, Thulliez P, Remington JS. Diagnosis of toxoplasmic encephalitis in patients with acquired immunodeficiency syndrome by using a new serologic method. J Clin Microbiol 1988; 26(12): 2541-3.

14. Brytting M, Xu W, Wahren B, Sundqvist VA. Cytomegalovirus DNA detection in sera from patients with active cytomegalovirus infections. J Clin Microbiol 1992; 30(8): 1937-41.

15. Preiksaitis JK, Brennan DC, Fishman J, Allen U. Canadian society of transplantation consensus workshop on cytomegalovirus management in solid organ transplantation final report. Am J Transplant 2005; 5(2): 218-27.

16. Gutierrez E, Hernandez E, Morales E, Praga M. Severe gastrointestinal involvement caused by late CMV: the importance of early treatment. Nefrologia 2007; 27(6): 779-80.

17. Perez-Valentin MA, Cofan F, Sole M, Llach J, Esforzado N, Campistol JM, et al. Atypical cytomegalovirus in renal transplantation: a new form of presentation. Nefrologia 2002; 22(4): 381-5.

18. Roberts TC, Brennan DC, Buller RS, Gaudreault-Keener M, Schnitzler MA, Sternhell KE, et al. Quantitative polymerase chain reaction to predict occurrence of symptomatic cytomegalovirus infection and assess response to ganciclovir therapy in renal transplant recipients. J Infect Dis 1998; 178(3): 626-35.

19. Ferreira-Gonzalez A, Fisher RA, Weymouth LA, Langley MR, Wolfe L, Wilkinson DS, et al. Clinical utility of a quantitative polymerase chain reaction for diagnosis of cytomegalovirus disease in solid organ transplant patients. Transplantation 1999; 68(7): 991-6.

20. Toyoda M, Carlos JB, Galera OA, Galfayan K, Zhang X, Sun Z, et al. Correlation of cytomegalovirus DNA levels with response to antiviral therapy in cardiac and renal allograft recipients. Transplantation 1997; 63(7): 957-63.
21. Emery VC, Sabin CA, Cope AV, Gor D, Hassan-Walker AF, Griffiths PD. Application of viral-load kinetics to identify patients who develop cytomegalovirus disease after transplantation. Lancet 2000; 355(9220): 2032-6.

22. Buchler M, Hurault de Ligny B, Madec C, Lebranchu Y. Induction therapy by anti-thymocyte globulin (rabbit) in renal transplantation: a 1-yr follow-up of safety and efficacy. Clin Transplant 2003; 17(6): 539-45.

23. Burke GW, 3rd, Kaufman DB, Millis JM, Gaber AO, Johnson CP, Sutherland DE, et al. Prospective, randomized trial of the effect of antibody induction in simultaneous pancreas and kidney transplantation: three-year results. Transplantation 2004; 77(8): 1269-75.

24. Delgado O,Sánchez A, Cervera M, Gascó J. La enfermedad por citomegalovirus en el trasplante renal. Farm Hosp 2000; 24(5): 296-301.

25. Deboni L, Manfro R, Reck P, Keitel E, Bittar A, Neumann J, et al. Incidence and impact of citomegalovirus infection and disease diagnosed by antigenemia in long-term surviral of renal transplant recipients. J Bras Nefrol 2002; 24(3): 115-26.

26. Sagedal S, Hartmann A, Rollag H. The impact of early cytomegalovirus infection and disease in renal transplant recipients. Clin Microbiol Infect 2005; 11(7): 518-30.

27. Hartmann A, Sagedal S, Hjelmesaeth J. The natural course of cytomegalovirus infection and disease in renal transplant recipients. Transplantation 2006; 82(2 Suppl): S15-7.

28. Baron C, Forconi C, Lebranchu Y. Revisiting the effects of CMV on long-term transplant outcome. Curr Opin Organ Transplant 2010; 15(4): 492-8.

29. Jassal SV, Roscoe JM, Zaltzman JS, Mazzulli T, Krajden M, Gadawski M, et al. Clinical practice guidelines: prevention of cytomegalovirus disease after renal transplantation. J Am Soc Nephrol 1998; 9(9): 1697-708.

30. Cardona N, Gaviria M, Uribe G, Jaramillo C. Anticuerpos contra los agentes del síndrome TORCH en el grupo de gestantes de Medellín-Colombia. Revista CES Medicina. 1987; 1(1): 27-31.

31. Correa M, Correa M, Ossa J, Builes M, Arbeláez M. Prevalencia de infección por citomegalovirus en receptores y donantes de trasplante renal en Medellín para 1988-1989. Acta Med Colomb 1990; 15(4): 175-9.

32. Correa M, Ossa J, García LF, Arbeláez M. Histocompatibilidad, citomegalovirus y trasplante renal. Acta Med Colomb 1991; 16(5): 235-43.

33. Correa M, Ossa J, Builes M,Arbeláez M. Interferón gama y factor necrosante de tumores/linfotoxina en el cultivo mixto de linfocitos de candidatos y donadores de trasplante renal. Acta Med Colomb 1992; 17(2): 103-7.

34. Schnitzler MA, Woodward RS, Brennan DC, Spitznagel EL, Dunagan WC, Bailey TC. Impact of cytomegalovirus serology on graft survival in living related kidney transplantation: implications for donor selection. Surgery 1997; 121(5): 563-8.

35. Schnitzler MA, Woodward RS, Brennan DC, Spitznagel EL, Dunagan WC, Bailey TC. The effects of cytomegalovirus serology on graft and recipient survival in cadaveric renal transplantation: implications for organ allocation. Am J Kidney Dis 1997; 29(3): 428-34.

36. Lowance D, Neumayer HH, Legendre CM, Squifflet JP, Kovarik J, Brennan PJ, et al. Valacyclovir for the prevention of cytomegalovirus disease after renal transplantation. International Valacyclovir Cytomegalovirus Prophylaxis Transplantation Study Group. N Engl J Med 1999; 340(19): 1462-70.

37. Resik Aguirre S, Enamorado Casanova A, Kourí Cardellá V, Suárez Morán C, García Infante S. Monitoreo de la infección por citomegalovirus en pacientes con trasplante renal: primera experiencia en Cuba. Revista Cubana de Medicina Tropical 2000; 52: 203-10.

38. Sagedal S, Nordal KP, Hartmann A, Degre M, Holter E, Foss A, et al. A prospective study of the natural course of cytomegalovirus infection and disease in renal allograft recipients. Transplantation 2000; 70(8): 1166-74.

39. Aranda V F, Alberú G J, Soto R LE, González A H, Muñoz T T, Mancilla U E, et al. Efectividad de la terapia anticipada con ganciclovir en receptores de trasplante renal de alto riesgo (R-/D+) para desarrollo de enfermedad por citomegalovirus. Revista de investigación clínica 2002; 54(3): 198-203.

40. Borroto Díaz G, Barceló Acosta M, Guerrero Díaz C. Infección por citomegalovirus en pacientes con injerto renal. Revista Cubana de Medicina. 2003; 42: 1.

41. Schnitzler MA, Lowell JA, Hardinger KL, Boxerman SB, Bailey TC, Brennan DC. The association of cytomegalovirus sero-pairing with outcomes and costs following cadaveric renal transplantation prior to the introduction of oral ganciclovir CMV prophylaxis. Am J Transplant 2003; 3(4): 445-51.

42. Sagedal S, Hartmann A, Nordal KP, Osnes K, Leivestad T, Foss A, et al. Impact of early cytomegalovirus infection and disease on long-term recipient and kidney graft survival. Kidney Int 2004; 66(1): 329-37.

43. Carstens J, Andersen HK, Spencer E, Madsen M. Cytomegalovirus infection in renal transplant recipients. Transpl Infect Dis 2006; 8(4): 203-12.

44. Khoury JA, Storch GA, Bohl DL, Schuessler RM, Torrence SM, Lockwood $\mathbf{M}$, et al. Prophylactic versus preemptive oral valganciclovir for the management 
of cytomegalovirus infection in adult renal transplant recipients. Am J Transplant 2006; 6(9): 2134-43.

45. Helantera I, Koskinen P, Finne P, Loginov R, Kyllonen L, Salmela K, et al. Persistent cytomegalovirus infection in kidney allografts is associated with inferior graft function and survival. Transpl Int 2006; 19(11): 893-900.

46. Ozdemir FN,Akgul A,Altunoglu A, Bilgic A, Arat Z, Haberal M. The association between cytomegalovirus infection and atherosclerotic events in renal transplant recipients. Transplant Proc 2007; 39(4): 990-2.

47. Guirado L, Rabella N, Diaz JM, Facundo C, Maderuelo A, Margall N, et al. Prophylactic and pre-emptive therapy for cytomegalovirus infection in kidney transplant patients using oral valganciclovir. Nefrologia 2008; 28(3): 293-300.

48. Abraham KA, O'Kelly P, Spencer S, Hickey DP, Conlon PJ, Walshe JJ. Effect of cytomegalovirus prophylaxis with acyclovir on renal transplant survival. Ren Fail 2008; 30(2): 141-6.

49. Hughes D, Hafferty J, Fulton L, Friend P, Devaney A, Loke J, et al. Donor and recipient CMV serostatus and antigenemia after renal transplantation: an analysis of 486 patients. J Clin Virol 2008; 41(2): 92-5.

50. San Juan R, Aguado JM, Lumbreras C, Fortun J, Munoz P, Gavalda J, et al. Impact of current transplantation management on the development of cytomegalovirus disease after renal transplantation. Clin Infect Dis 2008; 47(7): 875-82.

51. Corona-Nakamura AL, Monteon-Ramos FJ, Troyo-Sanroman R, Arias-Merino $\mathbf{M J}$, Anaya-Prado R. Incidence and predictive factors for cytomegalovirus infection in renal transplant recipients. Transplant Proc 2009; 41(6): 2412-5.

52. Kanter J, Pallardo L, Gavela E, Escudero V, Beltran S, Morales A, et al. Cytomegalovirus infection renal transplant recipients: risk factors and outcome. Transplant Proc 2009; 41(6): 2156-8.

53. Valenzuela M, Ortiz AM, Troncoso P, Vilches S. Strategies for prevention of cytomegalovirus infection in renal transplant patients. Transplant Proc 2009; 41(6): 2673-5.

54. Salazar E MP, Alba G A, Deluchi B A, Hunter M B, Godoy L J, Ferrario B M, et al. Infección y enfermedad por citomegalovirus en niños sometidos a trasplante de órgano sólido: Experiencia en un centro de referencia chileno. Rev Chil Infect 2009; 26: 311-7.

55. Taherimahmoudi M, Ahmadi H, Baradaran N, Montaser-Kouhsari L, Salem S, Mehrsai A, et al. Cytomegalovirus infection and disease following renal transplantation: preliminary report of incidence and potential risk factors. Transplant Proc 2009; 41(7): 2841-4.

56. Abate D, Saldan A, Fiscon M, Cofano S, Paciolla A, Furian L, et al. Evaluation of cytomegalovirus (CMV)-specific T cell immune reconstitution revealed that baseline antiviral immunity, prophylaxis, or preemptive therapy but not antithymocyte globulin treatment contribute to $\mathrm{CMV}$-specific $\mathrm{T}$ cell reconstitution in kidney transplant recipients. J Infect Dis 2010; 202(4): 585-94.

57. Eid AJ, Brown RA, Arthurs SK, Lahr BD, Eckel-Passow JE, Larson TS, et al. A prospective longitudinal analysis of cytomegalovirus (CMV)-specific CD4+ and CD8+ T cells in kidney allograft recipients at risk of CMV infection. Transpl Int 2010; 23(5): 506-13.

58. Weclawiak H, Kamar N, Mengelle C, Esposito L, Mohamed AO, Laurence $\mathbf{L}$, et al. Pre-emptive intravenous ganciclovir versus valganciclovir prophylaxis for de novo cytomegalovirus-seropositive kidney-transplant recipients. Transpl Int 2010; 23(10): 1056-64.

59. Browne BJ, Young JA, Dunn TB, Matas AJ. The impact of cytomegalovirus infection $>/=1$ year after primary renal transplantation. Clin Transplant 2010; 24(4): 572-7.

60. Li YT, Emery VC, Surah S, Jarmulowicz M, Sweny P, Kidd IM, et al. Extensive human cytomegalovirus (HCMV) genomic DNA in the renal tubular epithelium early after renal transplantation: Relationship with HCMV DNAemia and longterm graft function. J Med Virol 2010; 82(1): 85-93.

61. van de Berg PJ, Heutinck KM, Raabe R, Minnee RC, Young SL, van Donselaar-van der Pant KA, et al. Human cytomegalovirus induces systemic immune activation characterized by a type 1 cytokine signature. J Infect Dis 2010; 202(5): 690-9.

62. van der Beek MT, Berger SP, Vossen AC, van der Blij-de Brouwer CS, Press RR, de Fijter JW, et al. Preemptive versus sequential prophylactic-preemptive treatment regimens for cytomegalovirus in renal transplantation: comparison of treatment failure and antiviral resistance. Transplantation 2010; 89(3): 320-6.

63. Rizvi SA, Naqvi SA, Hussain Z, Hashmi A, Akhtar F, Hussain M, et al. Renal transplantation in developing countries. Kidney Int Suppl 2003; (83): S96-100.

64. Silva DdFLd, Gomes RHS, Moraes MMd, Medeiros RFLd, Santos ECdO, Jesus IMd. Perfil sorológico e molecular da infecção pelo citomegalovírus em pacientes transplantados de Belém-PA / Serological and molecular profiles of cytomegalovirus infection in transplant patients from Belém-PA. Cad Saúde Colet 2007; 15(3): 369-78

65. Zandberg M, de Maar EF, Hofker HS, Homan van der Heide JJ, Rosati S, van
Son WJ. Initial cytomegalovirus prophylaxis with ganciclovir: no guarantee for prevention of late serious manifestations of CMV after solid organ transplantation. Neth J Med 2005; 63(10): 408-12.

66. Soto S, Alcazar C, Jimeno L, Gonzalez Soriano MJ. Late-onset cytomegalovirus disease following renal transplantation: a report of two cases. Nefrologia 2007; 27(3): 391-2.

67. Rubin RH. The indirect effects of cytomegalovirus infection on the outcome of organ transplantation. JAMA 1989; 261(24): 3607-9.

68. Martin-Davila P, Fortun Abete J. Cytomegalovirus infection in kidney transplant patients: what is the best way to prevent it?. Nefrologia 2008; 28(3): 253-6.

69. Khameneh ZR. Occurrence of cytomegalovirus infection and factors causing reactivation of the infection among renal transplant recipients: a single center study. Saudi J Kidney Dis Transpl 2008; 19(1): 41-5.

70. Gomez E, Melon S, Fresno MF, Tricas L, Navascues R, de Ona M, et al. Remission of severe disease induced by CMV and lymphoproliferative post-transplant disease after changing the immunosuppressive regime. Nefrologia 2002; 22(6): 574-81.

71. Streblow DN, Orloff SL, Nelson JA. Acceleration of allograft failure by cytomegalovirus. Curr Opin Immunol 2007; 19(5): 577-82.

72. Toupance O, Bouedjoro-Camus MC, Carquin J, Novella JL, Lavaud S, Wynckel A, et al. Cytomegalovirus-related disease and risk of acute rejection in renal transplant recipients: a cohort study with case-control analyses. Transpl Int 2000; 13(6): 413-9.

73. Pouteil-Noble C, Ecochard R, Landrivon G, Donia-Maged A, Tardy JC, Bosshard S, et al. Cytomegalovirus infection--an etiological factor for rejection? A prospective study in 242 renal transplant patients. Transplantation 1993; 55(4): 851-7.

74. Li L, Chaudhuri A, Weintraub LA, Hsieh F, Shah S, Alexander S, et al. Subclinical cytomegalovirus and Epstein-Barr virus viremia are associated with adverse outcomes in pediatric renal transplantation. Pediatr Transplant 2007; 11(2): $187-95$.

75. Reischig T, Jindra P, Hes O, Bouda M, Kormunda S, Treska V. Effect of cytomegalovirus viremia on subclinical rejection or interstitial fibrosis and tubular atrophy in protocol biopsy at 3 months in renal allograft recipients managed by preemptive therapy or antiviral prophylaxis. Transplantation 2009; 87(3): 436-44.

76. Richardson WP, Colvin RB, Cheeseman SH, Tolkoff-Rubin NE, Herrin JT, Cosimi AB, et al. Glomerulopathy associated with cytomegalovirus viremia in renal allografts. $N$ Engl J Med 1981; (2): 57-63.

77. Herrera GA, Alexander RW, Cooley CF, Luke RG, Kelly DR, Curtis JJ, et al. Cytomegalovirus glomerulopathy: a controversial lesion. Kidney Int 1986; 29(3): 725-33

78. Streblow DN, Soderberg-Naucler C, Vieira J, Smith P, Wakabayashi E, Ruchti F, et al. The human cytomegalovirus chemokine receptor US28 mediates vascular smooth muscle cell migration. Cell 1999; 99(5): 511-20.

79. High KP. Atherosclerosis and infection due to Chlamydia pneumoniae or cytomegalovirus: weighing the evidence. Clin Infect Dis 1999; 28(4): 746-9.

80. Chiu B, Viira E, Tucker W, Fong IW. Chlamydia pneumoniae, cytomegalovirus, and herpes simplex virus in atherosclerosis of the carotid artery. Circulation 1997 96(7): 2144-8.

81. Campistol JM. Cardiovascular risk in patients with renal transplantation. Nefrologia 2002; 22 Suppl 4: 7-11.

82. Coronel F, Tornero F, Macía M, Herrero JA, Sánchez A, Barrientos A. Hiperlipidemia en el trasplante renal. Tratamiento con pantetina. Nefrologia 1995; 15(1): 68-73.

83. Kramer BK, Zulke C, Kammerl MC, Schmidt C, Hengstenberg C, Fischereder $\mathbf{M}$, et al. Cardiovascular risk factors and estimated risk for CAD in a randomized trial comparing calcineurin inhibitors in renal transplantation. Am J Transplant 2003; 3(8): 982-7.

84. Opelz G, Dohler B. Influence of immunosuppressive regimens on graft survival and secondary outcomes after kidney transplantation. Transplantation 2009; 87(6): 795-802.

85. Kasiske BL, Anjum S, Shah R, Skogen J, Kandaswamy C, Danielson B, et al. Hypertension after kidney transplantation. Am J Kidney Dis 2004; 43(6): 1071-81.

86. Opelz G, Dohler B. Improved long-term outcomes after renal transplantation associated with blood pressure control. Am J Transplant 2005; 5(11): 2725-31.

87. Vincenti F, Friman S, Scheuermann E, Rostaing L, Jenssen T, Campistol JM, et al. Results of an international, randomized trial comparing glucose metabolism disorders and outcome with cyclosporine versus tacrolimus. Am J Transplant 2007; 7(6): 1506-14.

88. Johnston O, Rose CL, Webster AC, Gill JS. Sirolimus is associated with newonset diabetes in kidney transplant recipients. J Am Soc Nephrol 2008; 19(7): $1411-8$. 
89. Marcen R. Immunosuppressive drugs in kidney transplantation: impact on patient survival, and incidence of cardiovascular disease, malignancy and infection. Drugs 2009; 69(16): 2227-43.

90. Schnitzler MA, Lowell JA, Hmiel SP, Hardinger KL, Liapis H, Ceriotti CS, et al. Cytomegalovirus disease after prophylaxis with oral ganciclovir in renal transplantation: the importance of HLA-DR matching. J Am Soc Nephrol 2003; 14(3): 780-5.

91. Paya C, Humar A, Dominguez E, Washburn K, Blumberg E, Alexander B, et al. Efficacy and safety of valganciclovir vs. oral ganciclovir for prevention of cytomegalovirus disease in solid organ transplant recipients. Am J Transplant 2004; 4(4): 611-20.

92. Boppana SB, Rivera LB, Fowler KB, Mach M, Britt WJ. Intrauterine transmission of cytomegalovirus to infants of women with preconceptional immunity. N Engl J Med 2001; 344(18): 1366-71.

93. Fowler KB, Stagno S, Pass RF, Britt WJ, Boll TJ, Alford CA. The outcome of congenital cytomegalovirus infection in relation to maternal antibody status. N Engl J Med 1992; 326(10): 663-7.

94. Griffiths PD. Chronic cytomegalovirus infection. J Virol Methods 1988; 21(14): 79-86.

95. Nemati E, Eizadi M, Lankarani MM, Kardavani B, Khoddami-Vishteh HR, Kalantar E, et al. Cytomegalovirus disease after kidney transplantation: clues to accurate diagnosis. Transplant Proc 2007; 39(4): 987-9.

96. Abbott KC, Hypolite IO, Viola R, Poropatich RK, Hshieh P, Cruess D, et al. Hospitalizations for cytomegalovirus disease after renal transplantation in the United States. Ann Epidemiol 2002; 12(6): 402-9.

97. Siu CW, Chan TM, Li FK, Choy BY, Lui SL, Lo CY, et al. Association between anti-thymocyte globulin administration and cytomegalic virus infection and/or CMV disease in cadaveric renal allograft recipients. Transplant Proc 2000; 32(7): 1932-4

98. Hibberd PL, Tolkoff-Rubin NE, Cosimi AB, Schooley RT, Isaacson D, Doran M, et al. Symptomatic cytomegalovirus disease in the cytomegalovirus antibody seropositive renal transplant recipient treated with OKT3. Transplantation 1992; 53(1): 68-72.

99. Oh CS, Stratta RJ, Fox BC, Sollinger HW, Belzer FO, Maki DG. Increased infections associated with the use of OKT3 for treatment of steroid-resistant rejection in renal transplantation. Transplantation 1988; 45(1): 68-73.

100. Margreiter R, Klempnauer J, Neuhaus P, Muehlbacher F, Boesmueller C, Calne RY. Alemtuzumab (Campath-1H) and tacrolimus monotherapy after renal transplantation: results of a prospective randomized trial. Am J Transplant 2008; 8(7): 1480-5.

101. Mycophenolate mofetil in cadaveric renal transplantation. US Renal Transplan Mycophenolate Mofetil Study Group. Am J Kidney Dis 1999; 34(2): 296-303.

102. Vitko S, Margreiter R, Weimar W, Dantal J, Kuypers D, Winkler M, et al. Three-year efficacy and safety results from a study of everolimus versus mycophenolate mofetil in de novo renal transplant patients. Am J Transplant 2005; 5(10): 2521-30.
103. Dmitrienko S, Yu A, Balshaw R, Shapiro RJ, Keown PA. The use of consensus guidelines for management of cytomegalovirus infection in renal transplantation. Kidney Int 2007; 72(8): 1014-22.

104. Opelz G, Dohler B, Ruhenstroth A. Cytomegalovirus prophylaxis and graft outcome in solid organ transplantation: a collaborative transplant study report. Am J Transplant 2004; 4(6): 928-36.

105. Strippoli GF, Hodson EM, Jones C, Craig JC. Preemptive treatment for cytomegalovirus viremia to prevent cytomegalovirus disease in solid organ transplant recipients. Transplantation 2006; 81(2): 139-45.

106. Kalil AC, Levitsky J, Lyden E, Stoner J, Freifeld AG. Meta-analysis: the efficacy of strategies to prevent organ disease by cytomegalovirus in solid organ transplant recipients. Ann Intern Med 2005; 143(12): 870-80.

107. Hodson EM, Jones CA, Webster AC, Strippoli GF, Barclay PG, Kable K, et al. Antiviral medications to prevent cytomegalovirus disease and early death in recipients of solid-organ transplants: a systematic review of randomised controlled trials. Lancet 2005; 365(9477): 2105-15.

108. Balfour HH, Jr., Chace BA, Stapleton JT, Simmons RL, Fryd DS. A randomized, placebo-controlled trial of oral acyclovir for the prevention of cytomegalovirus disease in recipients of renal allografts. N Engl J Med 1989; 320(21): 1381-7.

109. Kletzmayr J, Kotzmann H, Popow-Kraupp T, Kovarik J, Klauser R. Impact of high-dose oral acyclovir prophylaxis on cytomegalovirus (CMV) disease in CMV high-risk renal transplant recipients. J Am Soc Nephrol 1996; 7(2): 325-30.

110. Bailey TC. Prevention of cytomegalovirus disease. Semin Respir Infect 1993 8(3): 225-32.

111. Knight DA, Hejmanowski AQ, Dierksheide JE, Williams JW, Chong AS Waldman WJ. Inhibition of herpes simplex virus type 1 by the experimental immunosuppressive agent leflunomide. Transplantation 2001; 71(1): 170-4.

112. Reischig T, Jindra P, Hes O, Svecova M, Klaboch J, Treska V. Valacyclovir prophylaxis versus preemptive valganciclovir therapy to prevent cytomegalovirus disease after renal transplantation. Am J Transplant 2008; 8(1): 69-77.

113. Limaye AP, Corey L, Koelle DM, Davis CL, Boeckh M. Emergence of ganciclovir-resistant cytomegalovirus disease among recipients of solid-organ transplants. Lancet 2000; 356(9230): 645-9.

114. Kruger RM, Shannon WD, Arens MQ, Lynch JP, Storch GA, Trulock EP. The impact of ganciclovir-resistant cytomegalovirus infection after lung transplantation. Transplantation 1999; 68(9): 1272-9.

115. Bienvenu B, Thervet E, Bedrossian J, Scieux C, Mazeron MC, Thouvenot D, et al. Development of cytomegalovirus resistance to ganciclovir after oral maintenance treatment in a renal transplant recipient. Transplantation 2000; 69(1): $182-4$

116. Plotkin SA, Starr SE, Friedman HM, Brayman K, Harris S, Jackson S, et al. Effect of Towne live virus vaccine on cytomegalovirus disease after renal transplant. A controlled trial. Ann Intern Med 1991; 114(7): 525-31.

117. Marschall M, Stamminger T. Molecular targets for antiviral therapy of cytomegalovirus infections. Future Microbiol 2009; 4: 731-42. 